03

\title{
Влияние отверстий в пористой поверхности земли на парящий полет насекомого
}

\author{
(C) Xinping Zhou ${ }^{1,2}$, Yong Huang ${ }^{2,3}$ \\ ${ }^{1}$ School of Mechanical Science and Engineering, Huazhong University of Science and Technology, Wuhan, PR China \\ ${ }^{2}$ Department of Mechanics, Huazhong University of Science and Technology, Wuhan, PR China \\ ${ }^{3}$ Luoyang Ship Material Research Institute, Luoyang, PR China \\ E-mail: xpzhou08@hust.edu.cn
}

Поступило в Редакцию 31 марта 2021 г.

В окончательной редакции 31 марта 2021 г.

Принято к публикации 30 июля 2021 г.

Методом численного моделирования проведено исследование аэродинамики машущего крыла при низком числе Рейнольдса в непосредственной близости от поверхности земли с учетом пористости подстилающей поверхности. Примечательно, что вихревая дорожка создается как под влиянием поверхности земли и отверстий (пор) в ней, так и благодаря вращательному движению крыла. Обнаружено, что влияние отверстий выражается в уменьшении подъемной силы, при этом, если отверстия достаточно велики, они могут эффективно нейтрализовать эффект увеличения подъемной силы под влиянием земли, возникающий, когда крыло находится в непосредственной близости от ее поверхности. Предлагается эффективный метод оптимизации расчета полета в режиме машущего движения крыльев для робототехнических устройств, совершающих полеты над пористой поверхностью.

Ключевые слова: аэродинамика, машущее крыло, низкое число Рейнольдса, влияние поверхности земли, влияние отверстий.

DOI: 10.21883/PJTF.2021.22.51722.18796

Машущее движение крыльев в воздухе является чрезвычайно интересным элементом поведения летающих насекомых. В настоящее время все больший интерес вызывают научные разработки в области проектирования небольших по размерам робототехнических устройств с машущими крыльями (см., например, [1-3]). Взмахи крыльев обеспечивают движение летящего в воздухе насекомого и вверх, и вперед. Изучение аэродинамики процесса взмаха крыльями насекомого может быть полезным для понимания летательной способности насекомых и может быть использовано при проектировании робототехнических устройств на основе этих же принципов.

Исследования характеристик потоков воздуха вокруг крыла насекомого проводились и раньше. Факторам, влияющим на поведение насекомого в полете в непосредственной близости от земли, посвящены работы многих исследователей [4-8]. Было обнаружено, что земля оказывает значительное влияние на аэродинамические характеристики машущего крыла. Если говорить конкретно, существуют три стандартных режима изменения подъемной силы под влиянием земли, а именно увеличение силы, уменьшение силы и восстановление силы [5]. Изменяемая поверхность земли и неровности в виде цилиндров различного размера оказывают влияние, отличное от того, которое создается землей в обычном случае [8,9]. При изучении влияния на машущее крыло платформы конечных размеров было обнаружено, что аэродинамические характеристики процесса взмаха сильно зависят от положения крыла относительно края платформы. Далее было обнаружено, что вследствие воздействия платформы конечных размеров образуется обратная вихревая дорожка Кармана (von Kármán) [10]. В естественных условиях земля имеет пористую структуру, и в горизонтальной подстилающей поверхности, как и в поверхности земли, над которой осуществляется парение насекомого или робота, имитирующего насекомое, имеются отверстия (рис. 1). Так, например, пчелы машут крыльями, чтобы охладить пористые медовые соты. Некоторым насекомым свойственно парить вблизи лужаек (газонов) или пористых поверхностей. Считается, что наличие отверстий в горизонтальной пластине или в земле оказывает существенное влияние на аэродинамические характеристики машущего крыла. На данный момент известно совсем немного публикаций, посвященных проблеме влияния отверстий. В настоящей работе была разработана двумерная модель для исследования аэродинамики машущего крыла с учетом влияния отверстий в горизонтальной поверхности земли.

Маховые движения крыльев обычно имеют периодический характер и могут быть приблизительно описаны синусоидальной функцией. В настоящей работе рассматривается режим двумерного машущего движения крыла [3]. Уравнения, описывающие положение крыла 

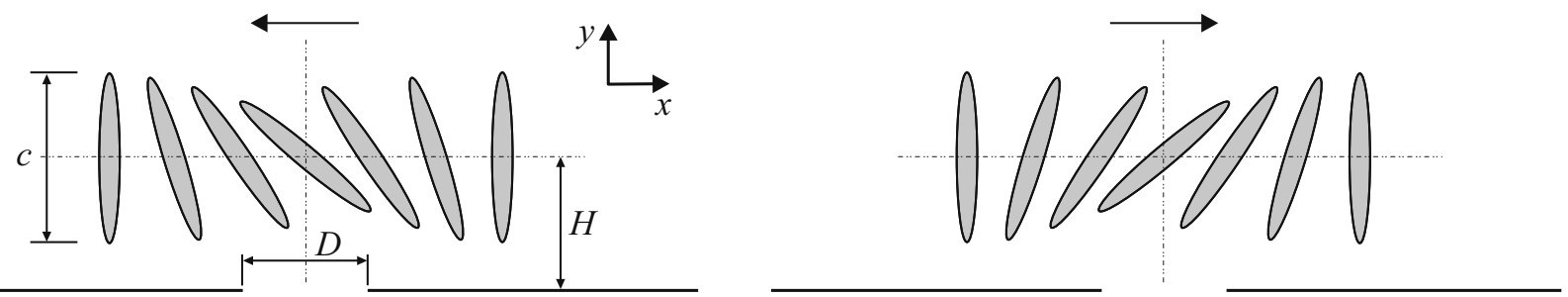

Рис. 1. Схематическое изображение взмаха крыла при парящем полете над горизонтальной поверхностью земли с отверстиями: слева - движение назад, справа - движение вперед. $c$ - длина хорды, $H$ - высота расположения крыла, т. е. расстояние между серединой хорды и поверхностью земли, $D$ - ширина отверстия.

при взмахе, имеют следующий вид:

$$
\begin{gathered}
x(t)=\frac{A}{2} \cos (2 \pi f t), \\
\alpha(t)=\alpha_{0}+\beta \sin (2 \pi f t+\phi),
\end{gathered}
$$

где $x(t)$ - положение центра несущей поверхности крыла, $A$ - амплитуда взмаха (т.е. расстояние между начальным положением при движении крыла вперед и начальным положением при движении крыла назад), $\alpha(t)$ - ориентация крыла относительно оси $x, \alpha_{0}-$ начальный угол атаки, равный $\pi / 2$ при симметричном движении, $\beta$ - амплитуда изменения угла атаки, $f-$ частота, $\phi$ - сдвиг фаз между $x(t)$ и $\alpha(t), t-$ время. Деформация крыла под воздействием воздушного потока здесь не учитывается.

В естественных условиях поток воздуха вокруг машущих крыльев насекомых в процессе полета всегда обладает небольшой скоростью и ламинарным характером. В настоящей работе нестационарное течение газа вблизи крыла рассматривается в модели несжимаемой жидкости и описывается системой, включающей уравнения Навье-Стокса и непрерывности:

$$
\begin{gathered}
\frac{\partial \mathbf{u}}{\partial t}+\mathbf{u} \cdot \nabla \mathbf{u}=-\frac{1}{\rho} \nabla p+v \nabla^{2} \mathbf{u}, \\
\nabla \cdot \mathbf{u}=0,
\end{gathered}
$$

где $\mathbf{u}-$ скорость движения воздуха, $p-$ статическое давление, $\rho$ - плотность воздуха $\left(1 \mathrm{~kg} / \mathrm{m}^{3}\right), v$ - кинематическая вязкость воздуха. Выражения для коэффициентов подъемной силы и лобового сопротивления имеют соответственно вид $C_{L}=\frac{F_{y}}{0.5 \rho U^{2} c}$ и $C_{D}=\frac{F_{x}}{0.5 \rho U^{2} c}$, где $F_{x}$ и $F_{y}$ - горизонтальная и вертикальная составляющие аэродинамической силы, действующей на крыло, $U$ скорость движения законцовки крыла.

В расчетах использовалась модель крыла в форме эллипса с длиной хорды $c=2.74 \mathrm{~mm}$ и отношением осей, равным 8. Начальный угол атаки принимался равным $90^{\circ}$, сдвиг фаз - 0, амплитуда взмаха - 2.8c. Двумерная круглая расчетная область состояла из небольшого внутреннего круга радиусом $4 c$ и большого внешнего кольца, внешний радиус которого составлял 20c. Расчетная сетка была построена с помощью программы
ANSYS ICEM CFD. Сетка во внутреннем блоке была более подробной, чем во внешнем, а наиболее плотная сетка использовалась вблизи поверхности крыла. Для описания движения крыла в соответствии с уравнениями (1) и (2) применяются пользовательские функции пакета ANSYS FLUENT [11]. В расчетах использовалась неявная схема для давления с расщеплением операторов (метод PISO - pressure implicit with splitting of operators). Для расчета давления использовалась схема PRESTO (pressure staggering option), а уравнение движения решалось с использованием противопоточной схемы второго порядка. После того как проведенный анализ показал независимость результатов от числа ячеек сетки и длины временно́го шага, были выбраны средний шаг сетки (101 220 ячеек) и средняя величина шага по времени $(T / 400)$. Правильность результатов моделирования была подтверждена путем их сравнения с данными работы [3].

Результаты численного моделирования показали, что по сравнению с маховыми движениями крыльев в свободном потоке влияние поверхности земли при $H / c=1-1.5$ приводит к увеличению осредненного по времени коэффициента подъемной силы. При значениях $H / c$ от 2 до 3 влияние земли проявляется в снижении подъемной силы. В случае же $H / c=3.5-5$ влияние земли выражается в восстановлении подъемной силы. Результаты хорошо согласуются с данными, представленными в литературе.

Эффект увеличения подъемной силы достигает максимума при $H / c=1$, при этом максимальный эффект увеличения подъемной силы становится намного сильнее, чем эффекты ее уменьшения и восстановления. Для исследования влияния отверстий на аэродинамические характеристики машущего крыла были выбраны различные значения величины $D / c$, а именно $K=D / c=0,1.5,2$ и 2.5, когда крыло находится в непосредственной близости от земли. На рис. 2 показано, как меняются коэффициенты подъемной силы и лобового сопротивления на протяжении одного периода при различных значениях $D / c$, а также представлен случай отверстия „бесконечной“ ширины (обозначаемого аббревиатурой KK, случай свободного течения) при $H / c=1$. На рис. 3 изображено вихревое поле на протяжении одного периода при $D / c=1$. Как видно из рис. 2 , при $H / c=1$ влияние отверстий приводит 
к резкому снижению пиков коэффициентов подъемной силы и лобового сопротивления. Причина заключается в том, что вихрь под нижней поверхностью крыла частично перетекает через отверстие (рис. 3). При этом в отверстии парный вихрь ослабляется, что и приводит к снижению пиков коэффициентов подъемной силы и лобового сопротивления. Кроме того, при увеличении размера отверстия его влияние становится меньше из-за ослабления влияния земли, что приводит к снижению эффекта увеличения подъемной силы. Интересно, что вращательное движение крыла приводит к образованию обратной вихревой дорожки Кармана под отверстием. Далее, если $D / c \geqslant 2.5$, значения коэффициентов подъемной силы и лобового сопротивления становятся близкими к значениям для случая КK, это означает, что отверстия достаточно большой ширины могут устранить сильное влияние поверхности земли.

Итак, вихревая дорожка образуется не только из-за вращательного движения крыла, но также из-за влияния как самой земли, так и отверстий. Если высота над землей достаточно мала, то под влиянием земли происходит увеличение подъемной силы. Наличие отверстий приводит к уменьшению коэффициентов подъемной силы и лобового сопротивления, что ослабляет эффект увеличения подъемной силы, возникающий под влиянием земли. В настоящей работе предлагается эффективный метод ослабления влияния земли на увеличение подъемной силы при расчете полета в режиме машущего движения крыльев, а также способы упрощения оптимизации
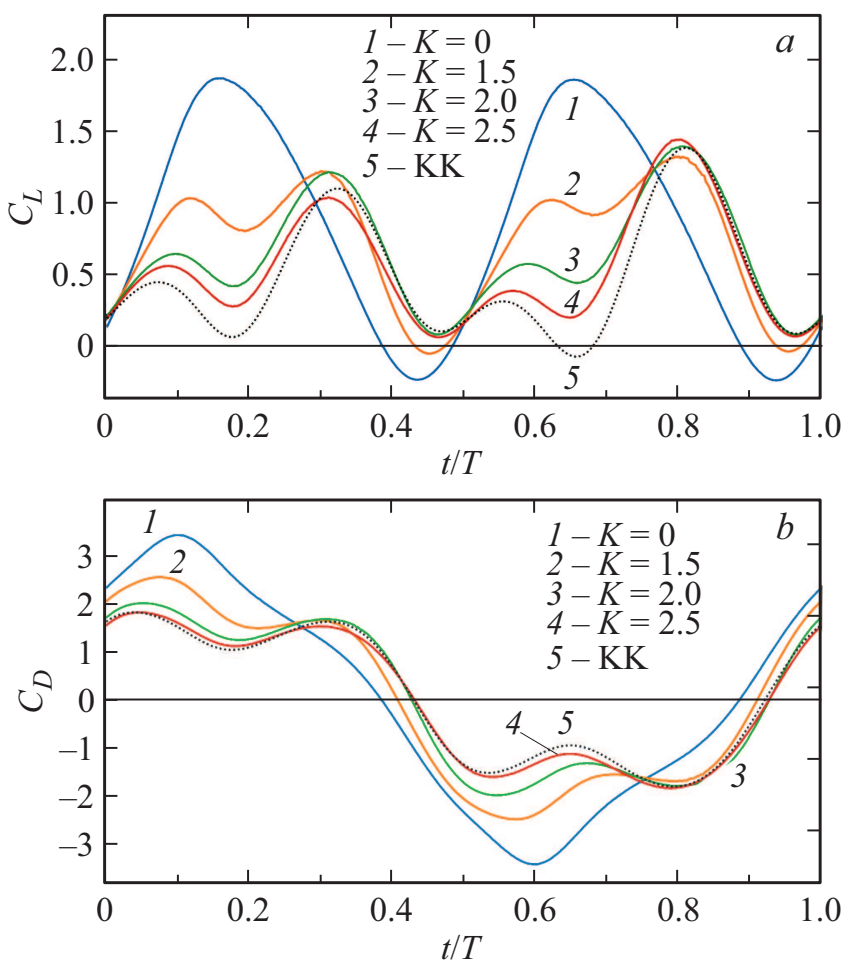

Рис. 2. Коэффициенты подъемной силы $(a)$ и лобового сопротивления $(b)$ при различных значениях $K=D / c . t-$ время, $T$ - период.
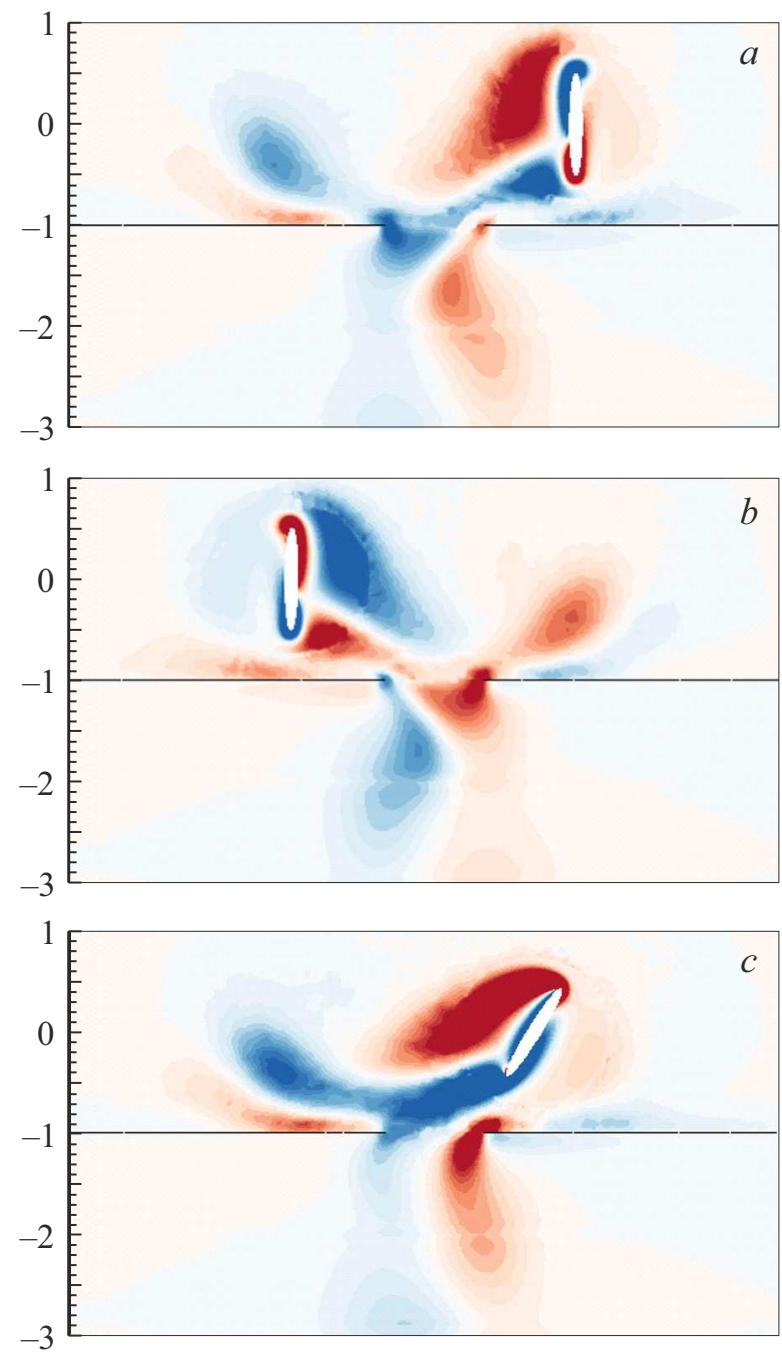

Рис. 3. Вихревое поле на протяжении одного периода при $K=D / c=1$ и $H / c=1$ для значений $t / T=0(a), 1 / 2(b)$ и $7 / 8(c) . t-$ время, $T-$ период.

расчетов полета в режиме машущего движения крыльев над пористой поверхностью.

\section{Конфликт интересов}

Авторы заявляют, что у них нет конфликта интересов.

\section{Список литературы}

[1] N.T. Jafferis, E.F. Helbling, M. Karpelson, R.J. Wood, Nature, 570, 491 (2019). DOI: 10.1038/s41586-019-1322-0

[2] H.V. Phan, H.C. Park, Science, 370, 1214 (2020). DOI: $10.1126 /$ science.abd3285

[3] Z.J. Wang, J.M. Birch, M.H. Dickinson, J. Exp. Biol., 207, 449 (2004). DOI: $10.1242 /$ jeb.00739

[4] J. Zerihan, X. Zhang, J. Aircraft, 37, 1058 (2000). DOI: $10.2514 / 2.2711$

[5] T. Gao, X. Lu, Phys. Fluids, 8, 87101 (2008). DOI: $10.1063 / 1.2958318$ 
[6] J. Wu, S.C. Yang, C. Shu, N. Zhao, W.W. Yan, J. Fluids Struct., 54, 247 (2015). DOI: 10.1016/j.jfluidstructs.2014.10.018

[7] T. Hagiwara, H. Takahashi, T. Takahata, I. Shimoyama, in IEEE 31th Int. Conf. on micro electro mechanical systems (MEMS 2018) (Belfast, Northern Ireland, UK, 2018), p. 832. DOI: 10.1109/MEMSYS.2018.8346684

[8] Q. Qu, P. Zuo, W. Wang, P. Liu, R.K. Agarwal, AIAA J., 53, 3144 (2015). DOI: 10.2514/1.J054155

[9] B. Yin, G. Yang, in Proc. of the ASME 2017 fluids engineering division summer meeting (Waikoloa, Hawaii, USA, 2017), vol. 1C, paper N FEDSM2017-69264. DOI: 10.1115/FEDSM2017-69264

[10] L. Wang, R.W. Yeung, Phys. Fluids, 28, 071902 (2016). DOI: $10.1063 / 1.4954656$

[11] ANSYS fluent theory guide, release 16.0 (ANSYS Inc., Canonsburg, Pennsylvania, 2015). 